Niniejsza publikacja jest dostępna na licencji Creative Commons. Uznanie autorstwa-Użycie niekomercyjne-Bez utworów zależnych 3.0 Polska. Pewne prawa zastrzeżone na rzecz autora. Zezwala się na wykorzystanie publikacji zgodnie z licencja - pod warunkiem zachowania niniejszej informacji licencyjnej oraz wskazania autora jako właściciela praw do tekstu. Treść licencji jest dostęna na stronie: http://creativecommons.org/licenses/by-nc-nd/3.0/pl/

Lingwistyka Stosowana 18: 3/2016, 105-111

\author{
Thorsten ROELCKE
}

Technische Universität Berlin

\title{
Kontrastive Fachsprachenlinguistik - eine funktionale Perspektive
}

\begin{abstract}
:
Contrastive LSP linguistics - a functional perspective

In the age of increasing internationalisation and globalisation, English has been established as lingua franca in science, technology and institutions. For this supremacy, good and less good reasons could be mentioned, as it has been discussed since the 20th century. In contrary, the advantages of multilinguialism and professsional communication in German are obvious (for instance in K. Ehlich 2006; C. Fandrych/ B. Sedlaczek 2012). In the context of this ongoing debate, German as a foreign language or better German as a foreign and special language has been mainly neglectet. The present article will not analyse the epistemic, ecological, social or political reasons for the usage of German as a foreign and special language in detail. Considering the plurality of German as a foreign and specialist language as problem related to foreign language teaching, the present article tries to find a solution for overcoming these difficulties and therewith supporting multilingualism in specialised communication. Therefore, the problem of pluralism itself will be outlined, followed by suggestions for solution processes based on a change of the linguistic perspective and an appropriate didactic approach.
\end{abstract}

\section{Vorbemerkungen}

Im Zeitalter zunehmender Internationalisierung und Globalisierung hat sich das Englische als internationale Lingua franca in Wissenschaft, Technik und Institutionen auch im deutschsprachigen Raum weitgehend etabliert. Für diese Vormachtstellung können viele gute - und auch weniger gute - Gründe angeführt werden. Probleme, die mit dem Englischen als internationaler Lingua franca verbunden sind, werden seit dem 20. Jh. diskutiert, wobei ihnen wiederholt diverse Vorteile fachlicher Mehrsprachigkeit im Allgemeinen wie auch Vorteile der fachlichen Kommunikation im Deutschen selbst gegenübergestellt werden (vgl. zum Beispiel K. Ehlich 2006; C. Fandrych/ B. Sedlaczek 2012). Im Zusammenhang mit dieser Diskussion hat der Bereich des Deutschen als Fremdsprache - oder besser: der Bereich des Deutschen als fachlicher Fremdsprache bislang jedoch nur wenig Berücksichtigung gefunden. Zwar mangelt es auch hier nicht an allgemeinen Begründungen für den Gebrauch des Deutschen neben anderen Sprachen, doch steht dabei die fachliche Kommunikation in deutscher Sprache meist im Hintergrund.

Auf die epistemischen, ökonomischen, sozialen oder politischen Gründe, die für den Gebrauch des Deutschen als fachlicher Fremdsprache sprechen, kann und soll an dieser Stelle nicht näher eingegangen werden. Im Folgenden wird mit der fachfremd- 
sprachlichen Pluralität vielmehr ein Problem aufgegriffen, das der Vermittlung des Deutschen als fachlicher Fremdsprache immer wieder im Wege steht und dessen Lösung einen wichtigen Beitrag zur fachkommunikativen Mehrsprachigkeit leisten kann. Dabei wird in Anlehnung an T. Roelcke (2015) zunächst das fachsprachliche Pluralitätsproblem selbst näher umrissen, um dann über einen Wechsel der linguistischen Perspektive und eine entsprechende didaktische Passung Lösungswege aus diesem Problem aufzuzeigen.

\section{Form und Funktion}

Das Pluralitätsproblem des Deutschen als fachlicher Fremdsprache in Wissenschaft, Technik und Institutionen lässt sich anhand von drei einander überlappenden Bereichen näher umreißen (vgl. Abb. 1):

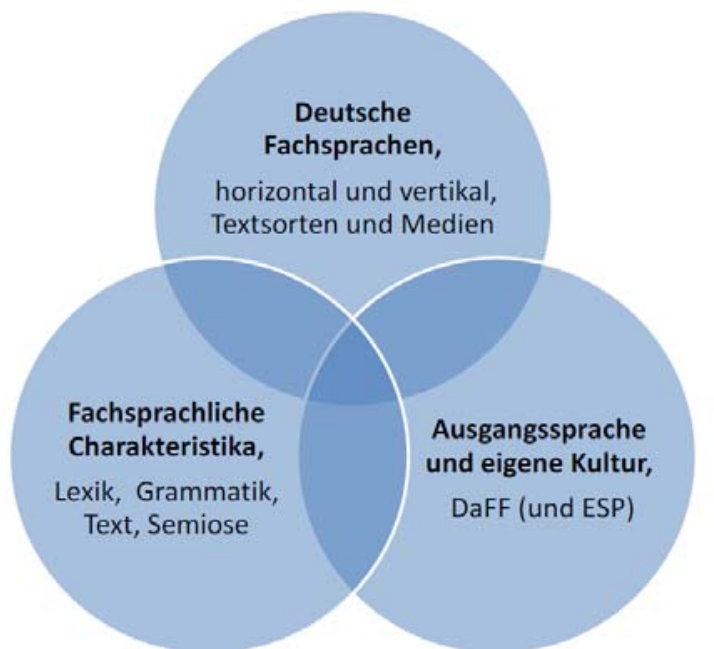

Abbildung 1: Pluralität des Deutschen als fachlicher Fremdsprache (nach T. Roelcke 2015: 16)

1. Die deutschen Fachsprachen differieren horizontal in verschiedenen Fächern bzw. Disziplinen und vertikal auf diversen Kommunikationsebenen; hinzu treten zahlreiche Textsorten und mediale Unterschiede.

2. Zahlreiche fachsprachliche Charakteristika bestehen sowohl auf lexikalischer als auch auf grammatischer und textueller Ebene; darüber hinaus finden sich Besonderheiten im Verhältnis zwischen Text und Bild.

3. Die Ausgangssprache der Lernenden steht in einer eigenen fachlichen Kultur, die sich von derjenigen des Deutschen (als Fremdsprache) unterscheidet; Analoges gilt für das Englische als Fach- bzw. Fachfremdsprache.

Die Literatur zu den Fachsprachen des Deutschen nähert sich dieser fachsprachlichen Pluralität in der Regel ausgehend von einzelnen sprachlichen Formen des Wortschatzes, der Grammatik oder des Textes. Dies gilt sowohl für primär linguistische Darstellungen wie diejenigen von H.-R. Fluck (1996), L. Hoffmann (1985) oder T. Roelcke (2010) als auch für primär didaktische, etwa von R. Buhlmann/ A. Fearns (2000), G. Graefen/ M. Moll 2011, J. Leisen 2010, U. Ohm/ C. Kuhn/ H. Funk (2007) oder G. Schade (2009). 
So tragfähig solche Zugänge aus fachsprachenlinguistischer Sicht also sicher auch sein mögen, so wenig zielführend erscheinen sie letztlich aus didaktischer Warte: Denn die Orientierung an der fachsprachlichen Form erlaubt: a) keine befriedigende Einsicht in die Gründe für die genannten formalen Besonderheiten; b) keine angemessene Erklärung für die fachsprachliche Pluralität im Deutschen; und c) keine hinreichende Verbindung mit der fachlichen Kultur der betreffenden Ausgangssprache.

Aus diesem Grund erscheint es sinnvoll, anstelle solcher formalen Charakteristika einen anderen Ausgangspunkt für linguistische und -didaktische Ansätze im Bereich des Deutschen als fachlicher Fremdsprache zu suchen.

Diesen Ausgangspunkt, der zwischen den zahlreichen sprachlichen Formen, den verschiedenen Fachsprachen des Deutschen sowie den unterschiedlichen Ausgangssprachen und deren Kulturen zu vermitteln vermag, bilden nun fachkommunikative Funktionen (vgl. auch T. Roelcke 2015: 18ff.): Diese bilden die Grundlage für die Wahl einzelner fachsprachlicher Formen sowie deren Gewichtung in einzelnen fachsprachlichen Varietäten und stehen dabei in Abhängigkeit von bestimmten fachlichen Kulturen und sprachlichen Strukturen des Deutschen und anderer Sprachen.

In der Forschungsliteratur zu deutschen Fachsprachen werden zahlreiche fachkommunikative Funktionen unterschieden - darunter auch:

- Differenzierung durch Erweiterung des lexikalischen Inventars;

- Erhöhung der Anonymität bei Steigerung der Objektivität;

- Erhöhung der Deutlichkeit;

- Steigerung von sprachlicher Ökonomie bzw. kommunikativer Effizienz.

Solchen fachkommunikativen Funktionen können nun zahlreiche fachsprachliche Formen zugeordnet werden - so zum Beispiel der Funktion der Differenzierung und Erweiterung des lexikalischen Inventars die formalen Ausprägungen „Definitionen“, „Entlehnungen“ und „Wortbildungen“ oder der Funktion der Erhöhung von Anonymität die Formen „Nominalstil“ und „Passivkonstruktionen“ (vgl. zur Übersicht auch Tab. 1).

\begin{tabular}{|c|c|c|c|c|c|}
\hline & $\begin{array}{l}\text { Erweiterung des } \\
\text { Inventars }\end{array}$ & $\begin{array}{l}\text { Erhöhung der } \\
\text { Anonymität }\end{array}$ & $\begin{array}{l}\text { Steigerung der } \\
\text { Objektivität }\end{array}$ & $\begin{array}{l}\text { Erhöhung der } \\
\text { Deutlichkeit }\end{array}$ & $\begin{array}{l}\text { Steigerung der } \\
\text { Ökonomie }\end{array}$ \\
\hline Abbildungen & & & $\mathrm{x}$ & $\mathrm{x}$ & $\mathrm{x}$ \\
\hline Attribute & & & & $\mathrm{x}$ & \\
\hline Definitionen & $x$ & & & $x$ & $x$ \\
\hline Entlehnungen & $\mathrm{x}$ & & & & \\
\hline Formeln & & & $\mathrm{x}$ & $\mathrm{x}$ & $x$ \\
\hline Kommentare & & & & $x$ & \\
\hline Konjunktionen & & & & $\mathrm{x}$ & \\
\hline Nominalstil & & $\mathrm{x}$ & $\mathrm{x}$ & & \\
\hline Passivformen & & $\mathrm{x}$ & $\mathrm{x}$ & & \\
\hline Tabellen & & & $\mathrm{x}$ & $\mathrm{x}$ & $\mathrm{x}$ \\
\hline Textgliederung & & & & $\mathrm{x}$ & $\mathrm{x}$ \\
\hline Wortbildungen & $\mathrm{x}$ & & & & $\mathrm{x}$ \\
\hline
\end{tabular}

Tabelle 1: Formen und Funktionen deutscher Fachsprachen (nach T. Roelcke 2015: 20) 


\section{Forschungsfragen}

An eine solche Zuordnung fachkommunikativer Funktionen und fachsprachlicher Formen schließen sich mindestens drei linguistische Fragestellungen an:

a) Welche fachkommunikativen Funktionen sind überhaupt zu unterscheiden? Dabei gilt es unter anderem auch zu klären, welche dieser Funktionen universell für Fachkommunikation als solche sind (etwa Deutlichkeit für einen bestimmten Expertenkreis), und welche spezifisch sind für bestimmte fachkommunikative Kulturen (so zum Beispiel Anonymität im westeuropäischen bzw. nordatlantischen Raum).

b) Welche sprachlichen Formen werden zur Erfüllung dieser Funktionen jeweils eingesetzt?

Diese Formen sind nicht universell, sondern hängen ihrerseits mehr oder weniger stark von den fachlichen Kulturen und sprachlichen Strukturen ab, in deren Rahmen sie verwendet werden (zu denken ist hier etwa an die Komposition als ein strukturelles Merkmal der deutschen Sprache im Hinblick auf die Erweiterung des lexikalischen Inventars, das im Chinesischen kein strukturelles Pendant findet).

c) Welche dieser Funktionen sind für einzelne Fächer, Ebenen, Textsorten und Medien jeweils charakteristisch?

Hiermit öffnet sich die Analyse kommunikativer Subkulturen und deren sprachlicher Formen. Da die Selektion der diversen Formen alleine nicht entscheidend ist, sondern auch deren Vorkommenshäufigkeit, ist hier im Weiteren auch mit Methoden der Quantitativen Linguistik zu arbeiten und eine funktionalquantitative Fachsprachenlinguistik zu entwickeln, die auch die Synergetik diverser Formen und verschiedener Funktionen analysiert.

Aus diesen allgemeinen sind nun weitere linguistische Fragestellungen aus einer kontrastiven Sicht abzuleiten. Im Folgenden werden drei genannt, wobei insbesondere das Deutsche als Fachfremdsprache berücksichtigt wird:

a) Welche (universellen oder spezifischen) Gemeinsamkeiten und Unterschiede zeigen die fachlichen Kulturen und fachkommunikativen Funktionen verschiedener Sprachgemeinschaften und deren Einzelsprachen?

Ein solcher Vergleich der fachlichen Kultur und deren kommunikativer Funktionen innerhalb des deutschen Sprachraums mit denjenigen verschiedener Ausgangssprachen bildet letztlich den Ausgangspunkt einer funktionalen Betrachtung des Deutschen als fachlicher Fremdsprache.

b) Wie werden einzelne fachkommunikative Funktionen in einzelnen Sprachen jeweils formal repräsentiert?

Der Blick auf funktionale und formale Gemeinsamkeiten und Unterschiede zwischen der Fachsprache im Deutschen und in anderen Sprachen hat sowohl kulturelle als auch strukturelle Gesichtspunkte zu berücksichtigen. Verschiedene fachsprachliche Formen für einander entsprechende fachkommunikative Funktionen erscheinen dabei von besonderem Interesse.

c) Welche Gemeinsamkeiten und Unterschiede zeigen die fachlichen Kulturen und fachkommunikativen Funktionen der diversen Einzelsprachen in ihrer spezifischen Differenzierung nach Fächern, Ebenen, Textsorten und Medien? 
Die funktional-quantitative Fachsprachenlinguistik steht hier angesichts einer variations- und textsortenabhängigen Synergetik fachsprachlicher Formen vor besonderen Herausforderungen, die zahlreiche Form/Funktion-Konstellationen des Deutschen (als fachlicher Fremdsprache) und diverser fachlicher Ausgangssprachen zu analysieren hat.

\section{Didaktische Passung}

Die zahlreichen Funktion/Form-Konstellationen innerhalb der deutschen und der anderen Fachsprachen ermöglichen eine hinreichende Differenzierung der Linguistik von Fachsprachen und erlauben somit eine passgerechte Didaktik des Deutschen als fachlicher Fremdsprache überhaupt. Eine solche didaktische „Passung“ des Deutschen als fachlicher Fremdsprache hat in drei Etappen zu erfolgen - und unter Berücksichtigung des Englischen als internationaler fachlicher Fremdsprache schließlich sogar in vier.

1. Die erste Etappe besteht in der Aufarbeitung der betreffenden fachlichen Ausgangssprache und deren Kultur. Dabei werden die fachkommunikativen Funktionen und die ihnen entsprechenden fachsprachlichen Formen erfasst und hinsichtlich der betreffenden horizontalen und vertikalen Variation sowie im Hinblick auf die einschlägigen Textsorten und Medien bestimmt.

2. In der zweiten Etappe werden die fachliche Kultur sowie die fachkommunikativen Funktionen und die diese repräsentierenden fachsprachlichen Formen des Deutschen unter Berücksichtigung ihrer horizontalen, vertikalen, textuellen und medialen Variation aufgearbeitet.

3. Auf den ersten beiden Etappen aufbauend werden schließlich in der dritten Etappe unter Berücksichtigung ihrer Variation die Gemeinsamkeiten und Unterschiede der fachlichen Kultur, der fachkommunikativen Funktionen und der fachsprachlichen Strukturen in der Ausgangssprache und im Deutschen erfasst. Damit wird der Lerngegenstand des Deutschen als fachlicher Fremdsprache differenziert aufgearbeitet und hinsichtlich des kommunikativen wie sprachlichen Bedarfs von einzelnen Lernenden präzise bestimmt. Diese Etappe bildet somit den Kern einer Didaktik des Deutschen als fachlicher Fremdsprache.

4. Im Zeitalter zunehmender internationaler Verflechtung erscheint der Erwerb des Deutschen als fachlicher Fremdsprache allein als nicht hinreichend. Daher wird hier eine vierte Etappe angesetzt, welche die kontrastive Aufarbeitung des Englischen als weiterer fachlicher Fremdsprache umfasst. Dabei gilt es, jeweils die fachlichen Kulturen, fachkommunikativen Funktionen und fachsprachlichen Formen der Ausgangssprache, des Deutschen und des Englischen unter Berücksichtigung ihrer Variation zu erfassen und miteinander zu vergleichen. Ein weiteres Argument für die Berücksichtigung des Englischen als fachlicher Fremdsprache ergibt sich aus dem Umstand, dass das Deutsche zunehmend als Tertiärsprache nach dem Englischen erlernt wird.

\section{Abschließende Bemerkungen}

Die Vielfalt fachsprachlicher Merkmale, die Variation innerhalb von Fachsprachen sowie der Kontrast zwischen Ausgangssprache und Deutsch als Fremdsprache stellen die Didaktik des Deutschen als fachlicher Fremdsprache vor ein Pluralitätsproblem. 
Traditionelle Ansätze der Linguistik und Didaktik werden diesem Problem in der Regel nicht gerecht, da sie meist an fachsprachlichen Formen ansetzen. Daher wird hier anregt, fachkommunikative Funktionen und Ausprägungen fachlicher Kultur zum Ausgangspunkt der linguistischen Analyse und der didaktischen Konzeption zu machen. Anhand von funktionalen Analysen deutscher und anderer Fachsprachen einschließlich ihrer Variation kann eine passgerechte Didaktik des Deutschen als fachlicher Fremdsprache begründet werden.

Eine solche funktionale Analyse von Fachsprachen ist bislang jedoch mehr Programm als Realität. Auch und gerade im Hinblick auf die weitere Entwicklung der Didaktik des Deutschen als fachlicher Fremdsprache öffnet sich hier ein wichtiges und umfangreiches Arbeitsfeld, das letztlich einen Beitrag zur Erhaltung und Förderung der fachsprachlichen Pluralität - auch und gerade unter Einschluss der deutschen Sprache - leisten kann.

Der vorliegende Beitrag trägt den Titel „Kontrastive Fachsprachenlinguistik - eine funktionale Perspektive“. Es sollte deutlich geworden sein, dass diese funktionale Perspektive auch und gerade aus fachsprachendidaktischer Sicht ein wichtiges Desideratum darstellt. Vor diesem Hintergrund hätte der Beitrag auch heißen können: „Kontrastive Fachsprachendidaktik - eine funktionale Perspektive“. Zu diskutieren wäre in diesem Falle dann, welche Funktion dem Sprachkontrast beim Erwerb des Deutschen als fachlicher Fremdsprache zukommt. Dabei ist davon auszugehen, dass gerade beim Erwerb von fachsprachlichen Kompetenzen neben der Förderung von Kommunikation auch der Reflexion von Sprache im Sprach- wie auch im Sach- bzw. Fachunterricht eine erhebliche Bedeutung zukommt (vgl. Abb. 2 und G. Kniffka/ T. Roelcke 2015).

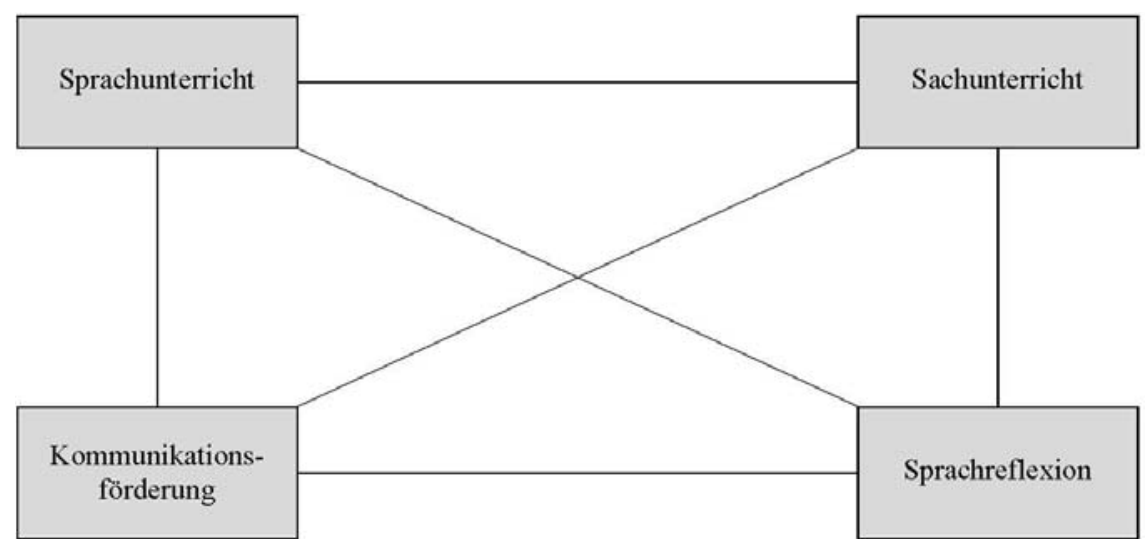

Abbildung 2: Fachliche und methodische Bereiche einer Didaktik des Deutschen als fachlicher Fremdsprache (nach T. Roelcke 2013).

\section{Bibliographie}

Buhlmann, R./ A. Fearns (2000), Handbuch des Fachsprachenunterrichts. Unter besonderer Berücksichtigung naturwissenschaftlich-technischer Fachsprachen, 6., überarbeitete und erweiterte Auflage. Tübingen. 
Ehlich, K. (2006), Mehrsprachigkeit in der Wissenschaftskommunikation - Illusion oder Notwendigkeit? In: K. Ehlich/ D. Heller (Hrsg.), Die Wissenschaft und ihre Sprachen. Bern etc., 17-38.

Fandrych, C./ B. Sedlaczek (2012), „I need German in my life“. Eine empirische Studie zur Sprachsituation in englischsprachigen Studiengängen in Deutschland. Tübingen

Fluck, H.-R. (1996), Fachsprachen. Einführung und Bibliographie. 5., überarbeitete und erweiterte Auflage. Tübingen/Basel.

Graefen, G./ M. Moll (2011), Wissenschaftssprache Deutsch: lesen - verstehen schreiben. Ein Lehr- und Arbeitsbuch. Frankfurt a.M. etc.

Hoffmann, L. (1985), Kommunikationsmittel Fachsprache. Eine Einführung. 2., völlig neu bearbeitete Auflage. Tübingen.

Kniffka, G./ T. Roelcke (2015), Fachsprachenvermittlung im Unterricht. Paderborn.

Leisen, J. (2010): Handbuch Sprachförderung im Fach. Bonn.

Ohm, U./ Ch. Kuhn/ H. Funk (2007), Sprachtraining für Fachunterricht und Beruf. Fachtexte knacken - mit Fachsprache arbeiten. Münster.

Roelcke, T. (2010), Fachsprachen. 3., neu bearbeitete Auflage. Berlin.

Roelcke, T. (2013), Fachsprachendidaktik in Haupt- und Realschulen - ein Weg der Ausbildungsvorbereitung? In: Ch. Efing (Hrsg.), Ausbildungsvorbereitung im Deutschunterricht der Sekundarstufe I. Die sprachlich-kommunikativen Facetten von „Ausbildungsfähigkeit“. (Text - Wissen - Kompetenz; 5). Frankfurt a.M. etc., 319-341.

Roelcke, T. (2015), Deutsch als fremde Wissenschaftssprache: Wege aus dem Pluralitätsproblem. In: A. Dohrn/ A. Kraft (Hrsg.), Fachsprache Deutsch - international und interdisziplinär. Hamburg, 15-30.

Schade, G. (2009), Einführung in die deutsche Sprache der Wissenschaften. Ein Lehrbuch für Ausländer. 13., neu bearbeitete und erweiterte Aufl. Berlin. 